\title{
Movilización pasiva continua en pacientes con artroplastia de rodilla
}

\author{
Leonardo Intelangelo," Diego Bordachar, ' Lisandro Nardin,, José L. Aparicio,, Raúl Beribé," Osvaldo Patiño* \\ "Unidad de Investigación Musculoesquelética, Centro Universitario de Asistencia, Docencia e Investigación - CUADI, \\ Universidad del Gran Rosario, Santa Fe, Argentina \\ ${ }^{*}$ Departamento de Rodilla, Sanatorio Mapaci, Rosario, Santa Fe, Argentina
}

\begin{abstract}
RESUMEN
Introducción: La artroplastia total de rodilla es el recurso terapéutico para pacientes con artrosis severa y gran incapacidad física. Sin embargo, muchos evolucionan con dolor y déficit funcional. En este estudio, se utiliza un tratamiento con movilización pasiva continua a partir de los 10 días de la cirugía. Materiales y Métodos: Se incluyó a 60 pacientes que fueron asignados, en forma aleatoria, a 2 grupos (30 en cada grupo). Al grupo 1 (G1, 23 mujeres) se le aplicó un protocolo de tratamiento convencional y, al grupo 2 (G2, 17 mujeres), el mismo programa y la adicción de un equipo de movimiento pasivo continuo a los 10 días de la intervención. Se evaluaron el dolor, la movilidad articular, la fuerza muscular y la función (WOMAC y prueba TUG). Resultados: No se observaron diferencias estadísticamente significativas en los parámetros estudiados, aunque sí una tendencia a la mejoría en el G2. En este grupo, la fuerza de extensión de la rodilla fue mayor y también hubo una correlación basal entre la fuerza y la prueba TUG. Conclusiones: El uso diferido de la movilización pasiva continua mejoró la fuerza de extensión de la rodilla y el rendimiento en la prueba TUG, aunque sin diferencias significativas entre ambos grupos. No se observaron efectos adversos.
\end{abstract}

Palabras clave: Movimiento pasivo continuo; artroplastia de rodilla; evaluación funcional; rodilla.

Nivel de Evidencia: I

\section{Continuous passive motion in knee arthroplasty patients}

\section{ABSTRACT}

Introduction: Total knee arthroplasty (TKA) is a valid therapeutic option for patients with severe arthritis and physical disability. However, many TKA patients develop pain and functional impairment. In our study, we used a continuous passive motion (CPM) device for exercise starting 10 days after surgery. Materials and Methods: The study population consisted of 60 patients, who were randomized into 2 groups. Group I (GI: 30 patients, 23 females) underwent the standard treatment and group II (GII, 30 patients, 17 females) underwent the standard treatment plus CPM starting 10 days after surgery. We evaluated pain, range of motion (ROM), extension muscle strength, and function (WOMAC and TUG tests). Results: All compared parameters yielded no statistically significant differences. A greater trend toward improvement was observed in Gll regarding some parameters: greater extension muscle strength and a baseline correlation between flexion strength and the TUG test. Conclusions: The use of CPM starting 10 days after of surgery improved the extension muscle strength and produced better TUG test results, although without any statistically significant difference with the standard procedure. No adverse effects were observed.

Key words: Continuous passive motion; knee arthroplasty; functional assessment; knee.

Level of Evidence: I

\section{INTRODUCCIÓN}

La artrosis de rodilla es un cuadro ortopédico común, y tiene una prevalencia general de artrosis sintomática que oscila entre el $8,1 \%$ y el $19 \% .{ }^{1-5} \mathrm{La}$ artroplastia total de rodilla (ATR) es el tratamiento de elección en pacientes con artrosis avanzada cuando las distintas opciones terapéuticas conservadoras han fracasado. ${ }^{6}$ El objetivo de la cirugía es obtener una articulación alineada y estable que alivie el dolor, incremente la movilidad y mejore la función. Sin embargo, el procedimiento requiere una extensa disección de partes blandas alrededor de la rodilla, que impone una importante agresión al mecanismo extensor y a otras estructuras periarticulares.

Recibido el 6-6-2019. Aceptado luego de la evaluación el 6-3-2020 • Lic. LEONARDO INTELANGELO • leonardo.intelangelo@ gmail.com http://orcid.org/0000-0002-2417-7914 Cómo citar este artículo: Intelangelo L, Bordachar D, Nardin L, Aparicio JL, Beribé R, Patiño O. Movilización pasiva continua en pacientes con artroplastia de rodilla. Rev Asoc Argent Ortop Traumatol 2020;85(3):234-245. https://doi.org/10.15417/issn.1852-7434.2020.85.3.998 
Sumado a esto, los cambios degenerativos crónicos, junto con la desalineación y la rigidez asociadas a la artrosis, también influyen en el resultado posoperatorio. ${ }^{7,8}$ Aunque un número considerable de pacientes refiere un alivio significativo y rápido del dolor, se ha comunicado que las limitaciones funcionales pueden persistir por períodos prolongados luego de la cirugía de reemplazo, especialmente cuando la discapacidad es evaluada mediante pruebas funcionales de desempeño. ${ }^{9-13}$ Ciertos factores, como la debilidad muscular posoperatoria, ${ }^{10,13}$ comprometen la movilidad general del paciente y afectan su desempeño en las actividades cotidianas. ${ }^{14}$

La movilización temprana se ha convertido en una parte importante de los protocolos de rehabilitación luego de la ATR, en contraposición a los protocolos clásicos que pregonaban la inmovilización en extensión para favorecer la cicatrización. ${ }^{15}$ El movimiento pasivo continuo (MPC) constituye un método de movilización posoperatoria proporcionado por un dispositivo que mueve la extremidad inferior, de forma lenta y continua, a través de una amplitud de movimiento preestablecida. El movimiento constante en flexión y extensión, y sin la carga impuesta por la actividad muscular, resulta en una oscilación sinusoidal en la presión intrarticular que crea un efecto de bombeo articular. ${ }^{16}$ Aunque se ha informado que el MPC puede mejorar la flexión, reducir el uso de analgésicos y la necesidad de manipulación bajo anestesia, ${ }^{15,17,18}$ varios estudios clínicos aleatorizados han concluido en que esta modalidad no ofrece un beneficio adicional cuando se utiliza un protocolo posoperatorio clásico basado en movilización articular y ejercicios activos. ${ }^{19-26}$

Uno de los principales factores que puede explicar la ausencia de beneficios clínicos con el uso del MPC es la metodología de aplicación, específicamente cuándo se inicia y cómo se indica. Siguiendo los principios propuestos por Salter, la mayoría de los estudios utilizó el MPC en el día 1 del posoperatorio. En esta etapa, la rodilla se encuentra bajo un intenso proceso inflamatorio como resultado de la cirugía de reemplazo, caracterizado por la acción de células inflamatorias que reaccionan para remover los tejidos dañados y formar el tejido de granulación sobre el cual se anclarán los fibroblastos durante la fase proliferativa para formar la nueva matriz de tejido conectivo. Por lo tanto, es posible que el movimiento precoz provoque alguna alteración del tejido cicatricial inicial incrementando el sangrado posquirúrgico. ${ }^{27-29}$ Esto es avalado por los hallazgos de Maniar y cols., quienes informaron que, en los pacientes que recibieron MPC, se observó que el edema posquirúrgico persistía más. ${ }^{19} \mathrm{La}$ forma de aplicación también varía mucho entre los estudios. El MPC es aplicado prácticamente, de manera exclusiva, durante la estancia hospitalaria, por un tiempo que oscila de $30 \min ^{19}$ a $6 \mathrm{~h},{ }^{20,22,24,25}$ y desde uno ${ }^{19}$ hasta 8 días. ${ }^{23}$ De hecho, en un estudio, el MPC se utilizó, de manera estacionaria, la primera noche después de la cirugía, manteniendo la rodilla flexionada a $90^{\circ}$ por 8-19 h, y se continuó con una modalidad dinámica de 5 h diarias, durante el resto de la estancia hospitalaria. ${ }^{26}$ Solo, en un estudio, el MPC continuó luego de la estancia hospitalaria, sumando un total de 18 días. ${ }^{21}$

Una alternativa factible a estas metodologías ineficaces podría ser aplicar el MPC de forma diferida. Esto tendría el potencial de reducir el sangrado posquirúrgico y, de esta manera, favorecer la resolución más rápida del proceso inflamatorio, reduciendo el dolor y permitiendo el movimiento pasivo durante la fase proliferativa de la reparación tisular. Por lo tanto, nuestro objetivo fue estudiar los efectos a corto plazo de la aplicación diferida de MPC utilizando un programa domiciliario intensivo.

\section{MATERIALES Y MÉTODOS}

\section{Participantes}

Este estudio prospectivo aleatorizado incluyó a 60 pacientes, de ambos sexos, de entre 55 y 75 años, con osteoartritis de rodilla, sometidos a una ATR unilateral. Los criterios de exclusión fueron: necesidad de cirugía de reemplazo por artritis inflamatoria, infección, artroplastia previa o tumores óseos, antecedente de osteotomía valguizante o varizante en la extremidad afectada o la contralateral, y antecedente de otra cirugía ortopédica mayor en la extremidad afectada y contralateral, y en la columna lumbar. Todos los pacientes firmaron el consentimiento informado y el estudio fue aprobado por el Comité de Ética de ambas instituciones.

\section{Aleatorización e intervenciones}

Los pacientes fueron asignados a uno de dos grupos. La aleatorización estuvo a cargo de uno de los investigadores (RB) siguiendo un código generado por el programa de aleatorización del sitio web www.randomizer.org.

A los pacientes del grupo 1 (G1) se les indicó un programa de ejercicios estandarizados dirigido a mejorar la movilidad articular y al fortalecimiento de los grupos musculares principales, que incluían abductores de cadera, flexores y extensores de rodilla, y extensores de tobillo. El programa debía comenzar tan pronto como el dolor lo permitiera luego del alta hospitalaria. Se instruyó a los pacientes a realizar los ejercicios de 2 a 4 veces por día, con una intensidad de 4 series de 8-12 repeticiones para cada ejercicio. 
A los pacientes del grupo 2 (G2) se les indicó el mismo programa de ejercicios, con la adición de un programa de MPC que comenzó a los 10 días del alta hospitalaria y se aplicó durante dos semanas. Un kinesiólogo con amplia experiencia en la aplicación clínica del dispositivo de MPC les enseñó a los pacientes cómo usarlo en el domicilio. Lo utilizaron $8 \mathrm{~h}$ por día, con intervalos de $2 \mathrm{~h}$ "on" y $2 \mathrm{~h}$ "off" (sumando un total de 4 intervalos de $2 \mathrm{~h}$ de aplicación diarios). El dispositivo fue programado para comenzar a mover la rodilla en el máximo rango de movilidad disponible de extensión y flexión, sin producir dolor y manteniendo, en todo momento, el contacto entre la extremidad y la superficie de apoyo. A partir del día 2, los pacientes debían incrementar el rango de flexión $5^{\circ}$ por día, respetando los mismos criterios. La velocidad de ejecución fue de $150 \%$ minuto.

\section{Procedimiento quirúrgico}

Todos los procedimientos de reemplazo se realizaron a través de un abordaje medial pararrotuliano. El protocolo de anestesia fue el mismo para ambos grupos. A todos se les administró anestesia espinal en combinación con inyección periarticular o bloqueo de nervio periférico (ciático, safeno o femoral). Se utilizó un implante Sigma ${ }^{\circledR}$ (DePuy Orthopaedics, Warsaw, IN, EE.UU.) en todos los procedimientos. Luego de la cirugía, se colocó un drenaje aspirativo por $24 \mathrm{~h}$. Al día siguiente, se permitió a los pacientes la carga de peso completa y la marcha por cortas distancias con la ayuda de un andador. El equipo médico indicó antinflamatorios no esteroides para aliviar el dolor posquirúrgico. No se administró ningún otro tipo de tratamiento analgésico.

\section{Evaluación de los pacientes}

Las evaluaciones estuvieron a cargo de dos kinesiólogos (LI, DB) y se realizaron 1-7 días antes de la cirugía (T1), y a los 30 (T2) y 90 días (T3) de la operación. En T1, se registraron los datos personales y la información adicional sobre el tiempo de evolución del dolor, y si el paciente practicaba o no actividad física, o recibía fisioterapia prequirúrgica. Las variables estudiadas en las tres instancias de evaluación fueron: intensidad del dolor, rango de movimiento de flexión y extensión, fuerza muscular y función.

La intensidad del dolor se evaluó usando la escala analógica visual. Se instruyó a los pacientes a establecer el nivel de dolor marcando en una línea de $10 \mathrm{~mm}$ (donde " 0 " indica ausencia de dolor y "10", el peor dolor imaginable) la intensidad promedio experimentada en las actividades diarias, durante los siete días anteriores a la evaluación. El rango de movimiento de flexión activa y de extensión de ambas rodillas se evaluó con un goniómetro universal. Para medir el rango de movimiento de flexión, se ubicó al paciente en decúbito prono, y se centró el goniómetro a nivel del epicóndilo lateral con la rama proximal apuntando hacia el trocánter mayor y la distal hacia el maléolo lateral. Se le solicitó que flexionara la rodilla mientras el evaluador controlaba las posibles compensaciones a nivel de la cadera. El déficit de extensión de la rodilla se evaluó con el paciente en posición supina. Se colocó el talón sobre un cilindro rígido y se le pidió al paciente que relajara la extremidad. El goniómetro se colocó de la misma manera y el déficit se registró con valores negativos. ${ }^{30}$

La fuerza muscular de ambas rodillas se midió con un dinamómetro manual (Lafayette modelo 01165, Lafayette Instrument Co., Lafayette, IN, EE.UU.). La fuerza de extensión y flexión de la rodilla se midió con el paciente sentado, con $90^{\circ}$ de flexión de caderas, $60^{\circ}$ de flexión de rodilla y los brazos cruzados en el pecho. ${ }^{31}$ Se colocó el pie sobre un banco pequeño para asegurar el ángulo correcto. El dinamómetro se apoyó a nivel del tercio distal de la pierna, sobre la cara anterior, para medir la fuerza de extensión y, sobre la cara posterior, para la fuerza de flexión. Se le pidió al paciente que empujara tan fuerte como le fuera posible contra la superficie del dinamómetro durante 6 segundos. Se realizaron tres repeticiones en cada dirección, separadas por 30 segundos de reposo, y se registró el valor promedio.

El desempeño funcional se evaluó mediante un cuestionario autoadministrado y una prueba de desempeño funcional. La versión Likert de la escala Western Ontario and McMaster Universities (WOMAC) es un cuestionario de 24 ítems que evalúa el dolor y las dificultades de la vida diaria, y su resultado oscila entre 0 (el mejor) y 96 (el peor).$^{32} \mathrm{El}$ rendimiento funcional se determinó con la prueba Timed-Up-and-Go (TUG). Esta prueba comienza con el paciente sentado en una silla de $46 \mathrm{~cm}$ de altura con apoyabrazos. A continuación, el paciente se levanta de la silla, camina $3 \mathrm{~m}$ hasta una línea en el suelo, retorna y vuelve a sentarse. El resultado registrado es el tiempo en segundos que toma completar la prueba. ${ }^{33}$

\section{Análisis estadístico}

Se llevaron a cabo dos tipos de análisis estadísticos (Statistica, Statsoft, EE.UU.), t de diferencias o t para datos apareados y factorial ANOVA para analizar los comportamientos de las curvas de datos de cada grupo. 


\section{RESULTADOS}

Los datos demográficos de la muestra se resumen en la Tabla. Todos los pacientes cumplieron con las tres etapas de evaluación. No se comunicaron complicaciones como resultado de las intervenciones. El protocolo domiciliario de MPC fue bien tolerado y tuvo una excelente adherencia.

Tabla. Datos demográficos de la muestra

\begin{tabular}{|l|c|c|}
\hline & Grupo 1 & Grupo 2 \\
\hline $\mathrm{n}$ & 30 & 30 \\
\hline Edad (años) & $69.76 \pm 6.79$ & $67.76 \pm 6.03$ \\
\hline Sexo femenino & 23 & 17 \\
\hline Tiempo de evolución (años) & $5.43 \pm 3.68$ & $4.91 \pm 3.75$ \\
\hline
\end{tabular}

Los valores se presentan como media (desviación estándar).

No se observaron diferencias significativas en la intensidad del dolor $(\mathrm{p}<0,001)$ y en la escala WOMAC ( $<0,001)$, entre ambos grupos, en T2 y T3. El rango de movimiento de extensión no mostró diferencias significativas en T2 y T3, en ambos grupos. El rango de movimiento de flexión de la pierna operada se redujo en ambos grupos, en T2, aunque la reducción fue menos pronunciada en los pacientes del G2 ( $<<0,001)$. Sin embargo, esta diferencia desapareció en T3 (Figura 1).

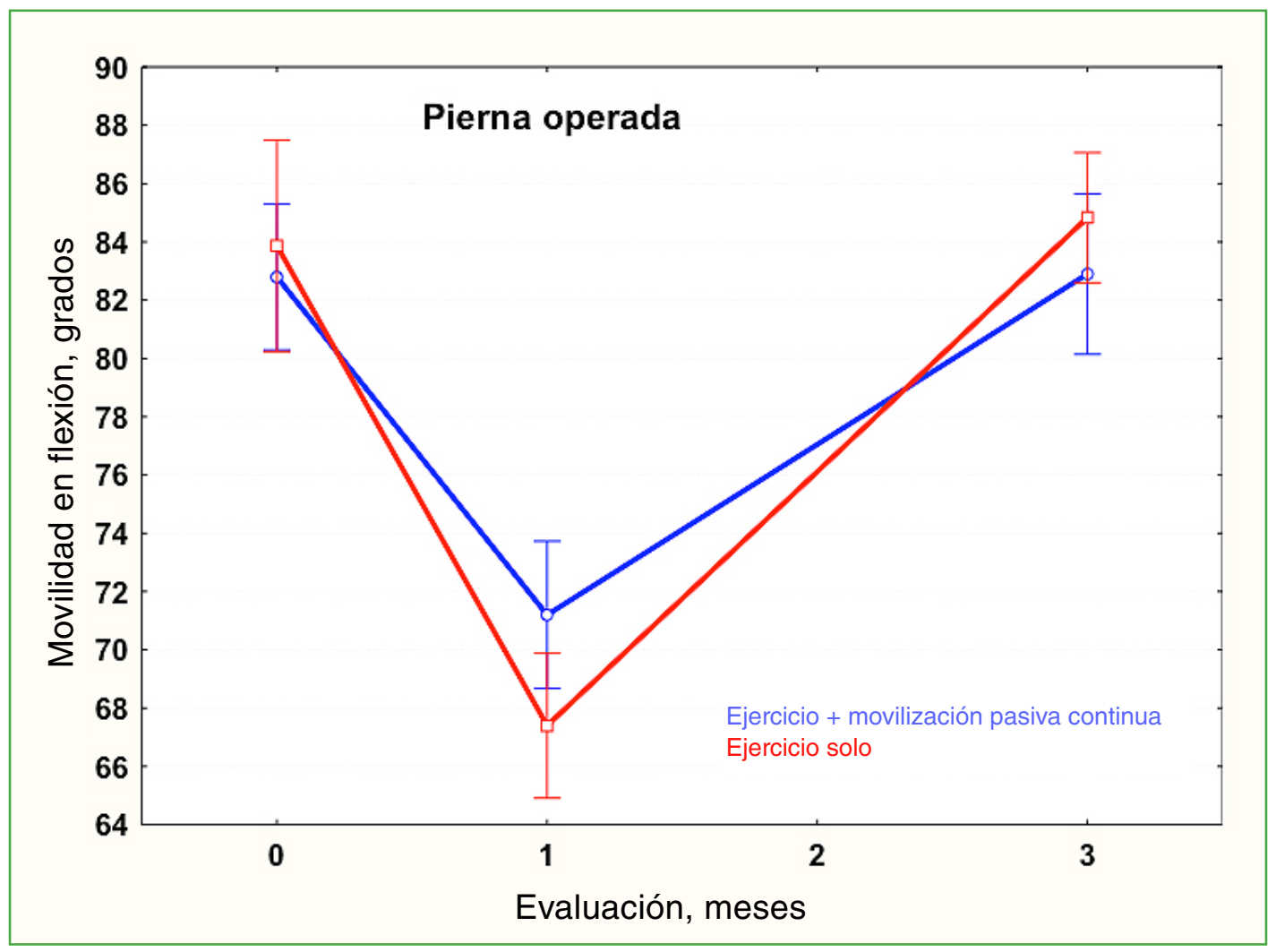

Figura 1. Movilidad en flexión de la pierna operada (expresada en grados). 
En ambos grupos, la fuerza de extensión de la rodilla de la pierna operada disminuyó en T2 y aumentó en T3. Los pacientes del G2 mostraron una tendencia clara, aunque no significativa, a una menor disminución en la fuerza en T2, manteniendo esa posible diferencia en T3 (Figura 2).

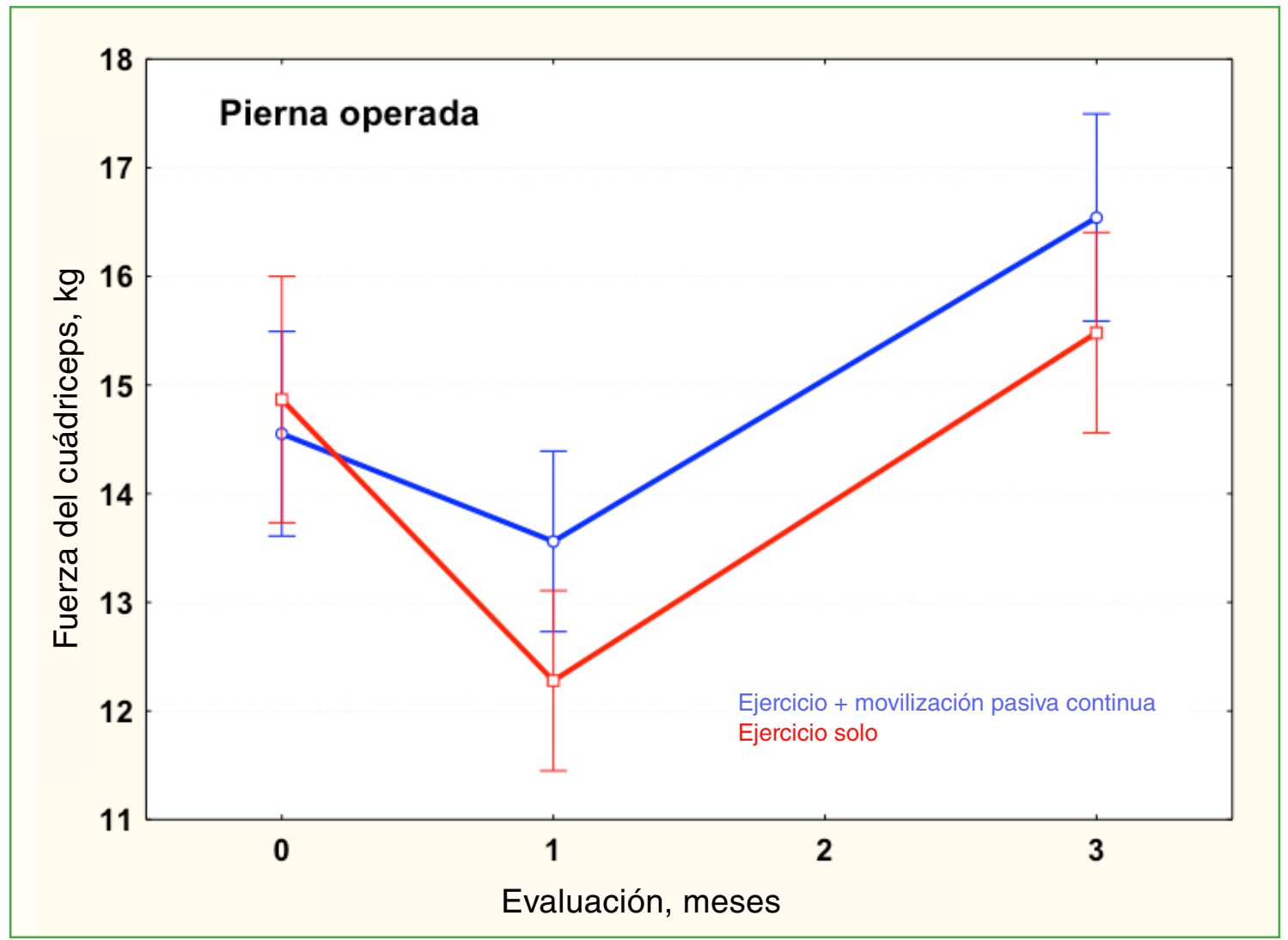

Figura 2. Fuerza de extensión de la pierna operada (expresada en kilogramos).

La fuerza de flexión de la pierna operada se incrementó en T2 y T3, en ambos grupos, la diferencia fue significativa solo en T3 (ANOVA p <0,02) (Figura 3).

Sorpresivamente, los valores de la prueba TUG fueron más altos en T1 y, en general, en el G2. En T2, los valores de la prueba TUG estaban significativamente incrementados en ambos grupos, pero el aumento fue estadísticamente significativo solo en el G2 ( $p>0,004)$. En T3, el tiempo de la prueba disminuyó claramente en ambos grupos y arrojó valores inferiores a los de la evaluación preoperatoria, aunque se observó una tendencia a una mayor disminución en el G2 (Figura 4). 


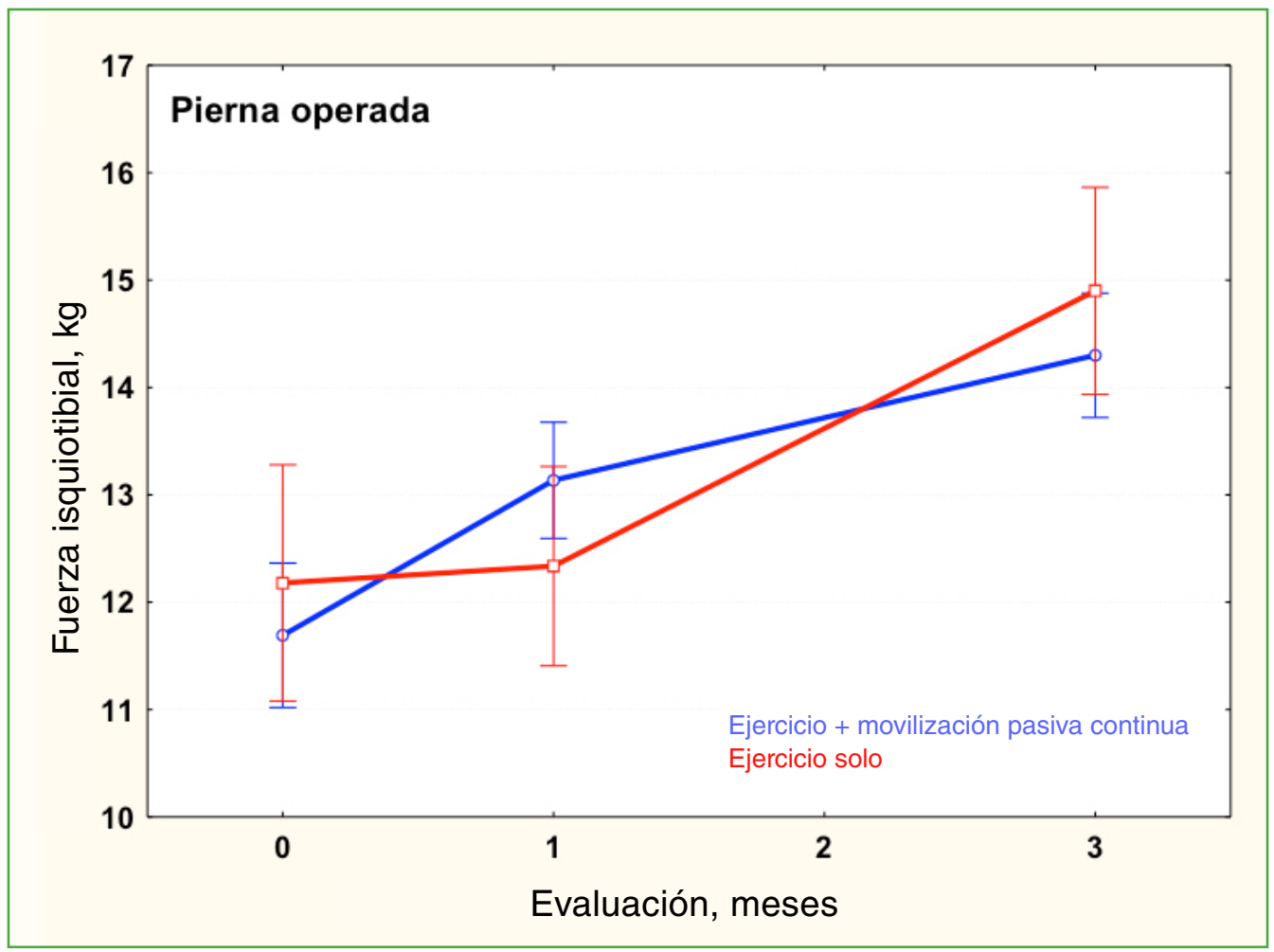

Figura 3. Fuerza de flexión de la pierna operada (expresada en kilogramos).

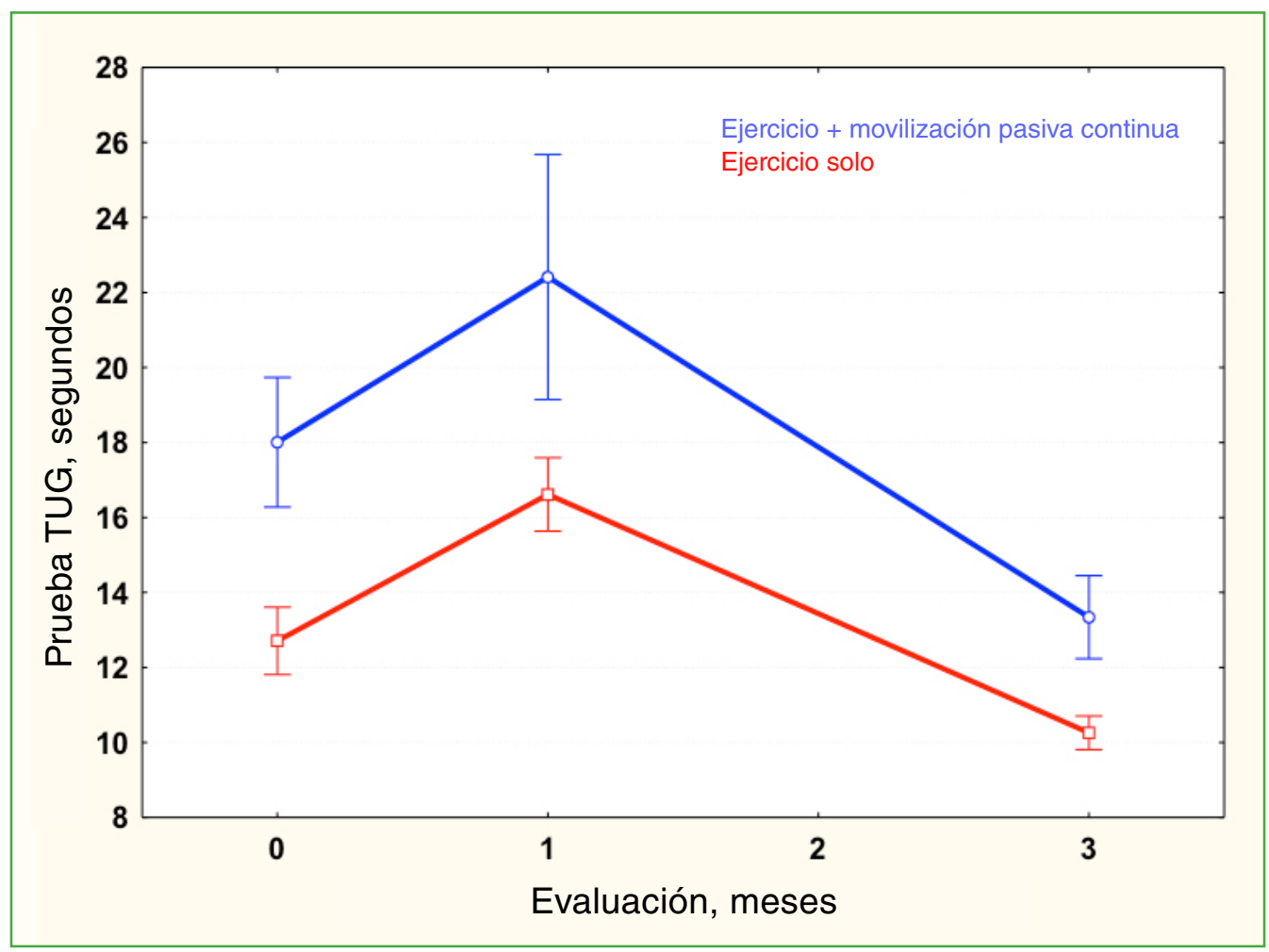

Figura 4. Valores de la prueba Timed-Up-and-Go (TUG) para el grupo 1 y el grupo 2 (expresados en segundos). 
En el G1, la fuerza de extensión de la rodilla de la pierna operada y de la no operada no mostró correlación con el resultado de la prueba TUG. Por el contrario, existió una correlación consistente entre la fuerza de extensión de la rodilla y la prueba TUG en todas las fases de evaluación en la pierna operada de los pacientes del G2 (p <0,001). Los análisis secundarios mostraron que, en el G2, tanto para la pierna operada como para la no operada, alcanzar una fuerza de extensión de la rodilla de $23,5 \mathrm{~kg}$ a los 90 días (T3) garantizó un resultado inferior a 12 segundos en la prueba TUG (Figura 5). Hubo también una correlación basal entre la fuerza de flexión de la rodilla y la prueba TUG, que se perdió en T2 y apareció nuevamente en T3 solo en el G2 (Figura 6). Curiosamente, se observó una tendencia similar en la pierna no operada.

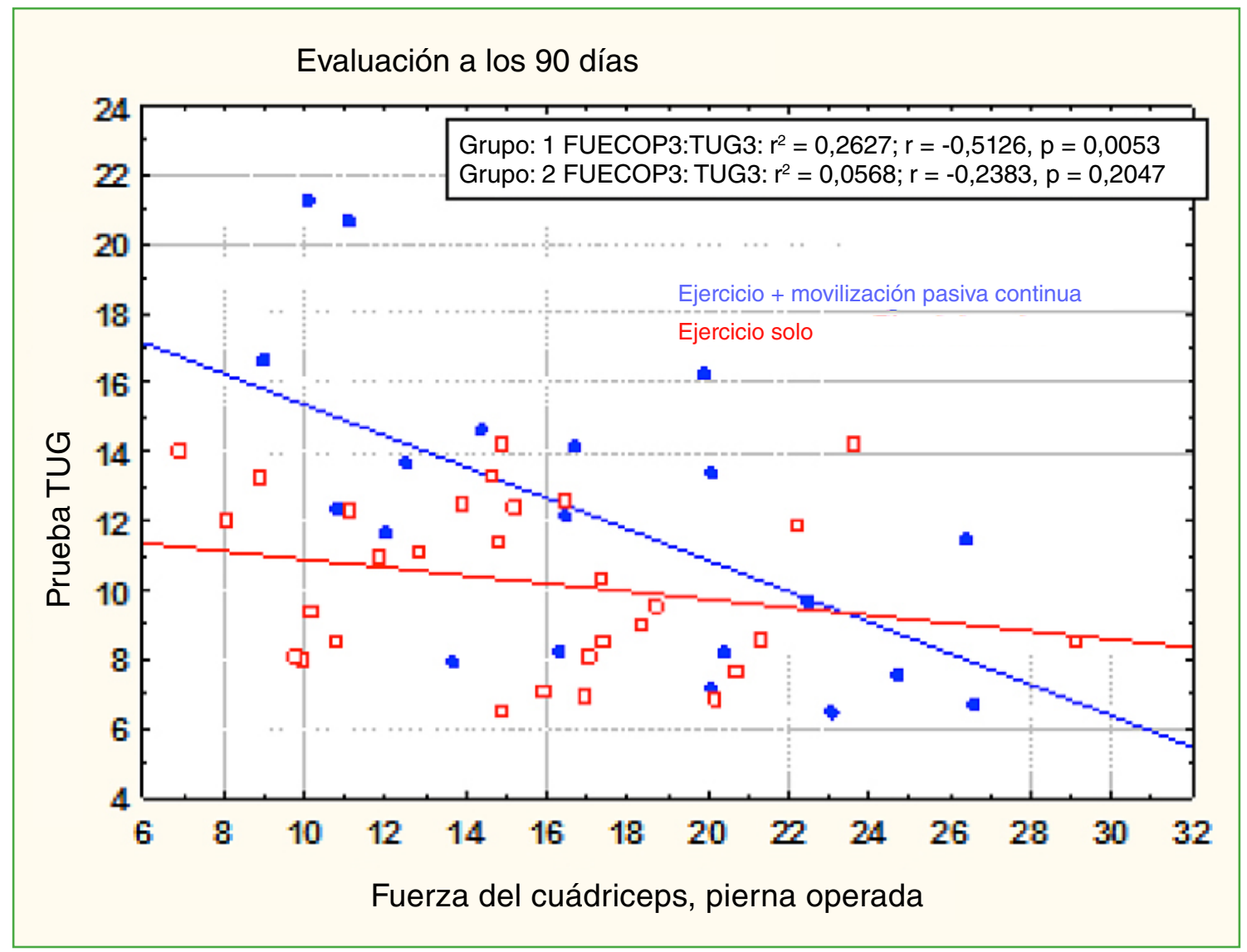

Figura 5. Correlación entre la prueba Timed-Up-and-Go (TUG) y la fuerza del cuádriceps para el grupo 1 y el grupo 2, a los 90 días de la cirugía. 


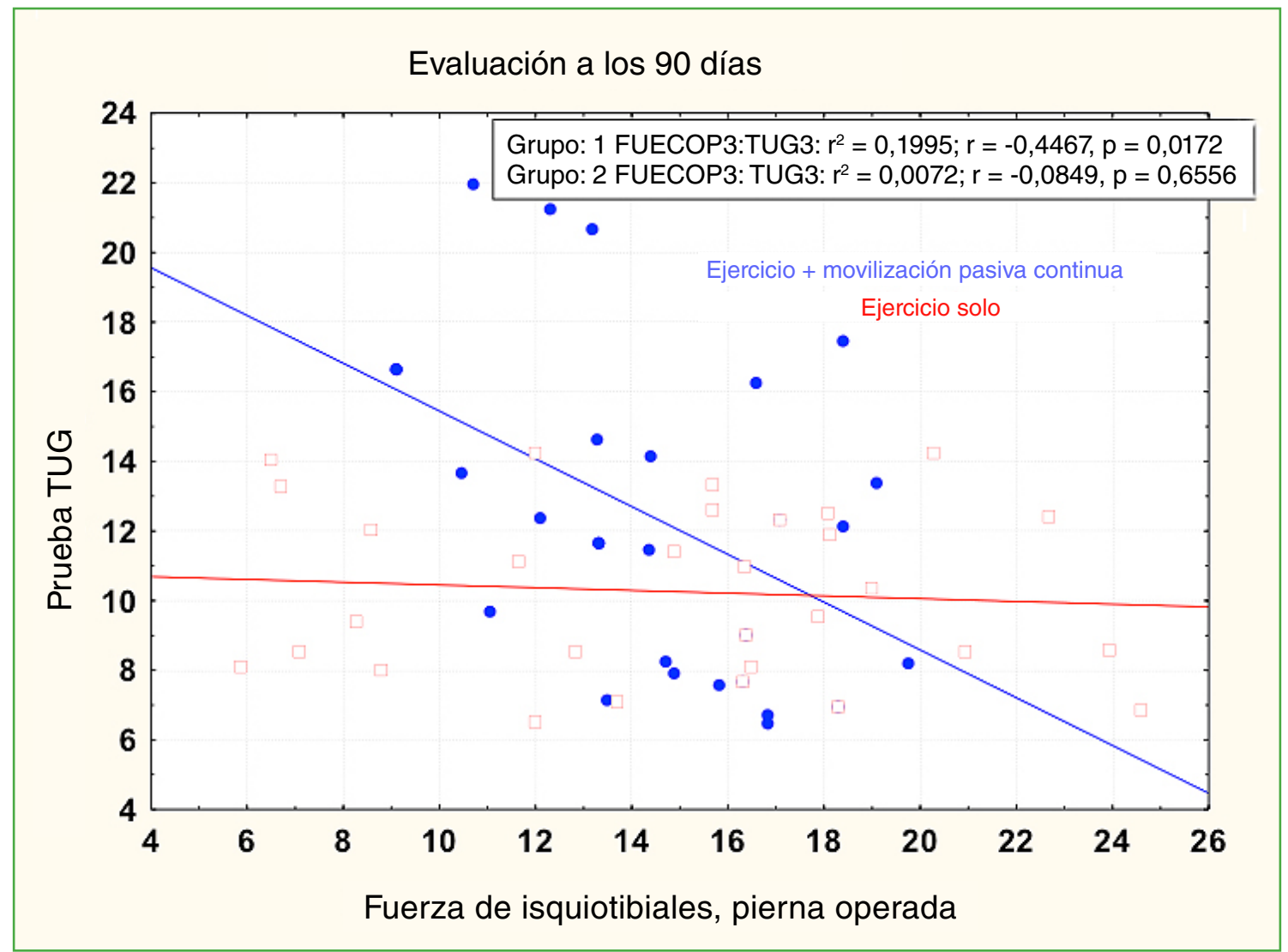

Figura 6. Correlación entre la prueba Timed-Up-and-Go (TUG) y la fuerza de isquiotibiales para el grupo 1 y el grupo 2, a los 90 días de la cirugía.

\section{DISCUSIÓN}

La mayoría de los estudios que evaluaron los efectos de la aplicación inmediata del MPC luego de una ATR no han reportado beneficios adicionales cuando se lo comparó con un programa convencional de terapia física. En este estudio, se propuso un nuevo protocolo de MPC que consistió en un programa intensivo y puramente domiciliario de 10 días, sin la aplicación durante la estancia hospitalaria. Los resultados mostraron que la adición de dicho protocolo se asoció a mejoras significativas en la prueba TUG y la fuerza de extensión y flexión de la rodilla, sin beneficios agregados en el dolor, el rango de movimiento y la escala WOMAC.

La fase final de la artrosis de rodilla se caracteriza por dolor resistente y limitación del rango de movimiento. La limitación dolorosa de las actividades cotidianas constituye la principal indicación de la ATR. Incrementar el rango de movimiento y contribuir al alivio del dolor son los principales objetivos perseguidos en la aplicación del MPC luego de la cirugía de reemplazo. Sin embargo, tal como lo publican otros autores, en nuestro estudio, no se hallaron diferencias significativas al compararlo con un programa estandarizado de ejercicios. Esto puede explicarse por los cambios que se producen a nivel del hueso subcondral y el cartílago articular. Con el avance de la enfermedad, el incremento de las cargas a nivel del hueso subcondral dispara un proceso de remodelación ósea asociado a un fenómeno de angiogénesis. Los vasos neoformados y los nervios sensoriales asociados son conducidos a través de canales vasculares hacia el cartílago, proporcionando a este tejido, originariamente aneural, las facultades de nocicepción y sensibilización periférica. ${ }^{34,35}$ Por consiguiente, es probable que la resección de estas estructuras hipersensibles sea el factor principal en el alivio del dolor posoperatorio. Además, los avances generados en los procedimientos de balanceo de partes blandas y alineación de los componentes protésicos con la cirugía asistida por navegación pueden justificar la ausencia de beneficios adicionales con el uso del MPC. Se necesitan futuros estudios que evalúen los efectos de este protocolo comparando pacientes con diversos niveles de compromiso preoperatorio de la movilidad, para mejorar la indicación del MPC luego de la ATR. 
La evaluación objetiva de la función en pacientes con reemplazo de rodilla es otra medición de gran interés clínico. Para dicho propósito, existen dos tipos de herramientas: los cuestionarios autoadministrados y las pruebas de rendimiento funcional. Se ha reportado que las escalas funcionales fallan en detectar los déficits funcionales reales luego de la ATR, principalmente, porque el resultado está muy influenciado por la percepción de dolor. ${ }^{10}$ Por el contrario, se ha demostrado que las pruebas funcionales muestran una relación consistente y directa con la fuerza del cuádriceps, y una pobre correlación con el dolor y las escalas autoadministradas, ${ }^{10,36,37}$ y su valor predictivo es importante. ${ }^{38}$ Por consiguiente, la evaluación funcional luego de la ATR debe incluir ambos tipos de mediciones. Según nuestro conocimiento, solo pocos estudios han incluido pruebas de rendimiento funcional como una medición del resultado. En tres estudios, el MPC fue aplicado en la sala de recuperación y no se observaron mejoras. ${ }^{19,22,23}$ Como se describió antes, la aplicación inmediata del MPC luego de la cirugía incrementa el sangrado posquirúrgico y favorece la perpetuación del edema. En consecuencia, es posible que esta intervención prolongue, en lugar de revertir, la inhibición muscular artrogénica, una causa conocida de debilidad de extensión de la rodilla preoperatoria y posoperatoria. ${ }^{39-41}$ En este estudio, la fuerza de extensión de la rodilla disminuyó en T2, en ambos grupos, pero se observó una tendencia a una menor disminución en los pacientes que recibieron MPC, y que esa diferencia se mantuvo en T3. Se podría argumentar que la aplicación diferida de MPC brinda a la rodilla un tiempo de enfriamiento, facilitando la posterior remoción del edema sin un estrés adicional sobre la articulación y los tejidos blandos. De acuerdo con esto, se halló que, solo en el G2, la fuerza de extensión de ambas rodillas se correlacionó con el resultado de la prueba TUG en T2 y T3, esto indica un efecto del MPC sobre la extremidad contralateral. ${ }^{40,41}$ Herbold y cols. también utilizaron la prueba TUG, pero solo los pacientes con pobre flexión posoperatoria de rodilla $\left(45-75^{\circ}\right)$ fueron incluidos en su estudio, y la aplicación del MPC comenzó a los cinco días de la cirugía, de manera que no pueden realizarse comparaciones directas. ${ }^{42}$ Se necesitan estudios para establecer conclusiones definitivas sobre los efectos del MPC en el rendimiento funcional.

Este estudio tiene algunas limitaciones. Primero, el tamaño de la muestra puede haber influenciado la potencia de los resultados. Los próximos estudios deberían evaluar los efectos de este protocolo en una muestra más grande de pacientes para confirmar los resultados obtenidos en esta investigación. La segunda limitación puede ser el tiempo de seguimiento. El tiempo fue elegido en función de los reportes de varios autores que indican que el MPC produce solo efectos a corto plazo. Si el efecto del MPC sobre el rendimiento funcional se confirma en estudios futuros, la intervención podría estar justificada si el paciente desea retornar más rápidamente a sus actividades cotidianas. Por último, este estudio no analizó la influencia de factores, como el índice de masa corporal y el ángulo de valgo preoperatorio, sobre el resultado final.

\section{CONCLUSIONES}

Se presentó un nuevo protocolo de MPC que consiste en un programa domiciliario intensivo a partir de los 10 días de la ATR. Los resultados mostraron que la adición de este protocolo de MPC a un programa convencional de terapia física incrementó más la fuerza de extensión de la rodilla y mejoró el rendimiento en la prueba TUG. Estos resultados podrían atribuirse al efecto del MPC sobre la remoción del edema posquirúrgico y la disminución de la inhibición muscular artrogénica. Se necesitan futuros estudios que evalúen este protocolo en una muestra más grande de pacientes para confirmar los resultados.

Conflicto de intereses: Los autores no declaran conflictos de intereses.

ORCID de D. Bordachar: https://orcid.org/0000-0003-3741-4138

ORCID de L. Nardin: https://orcid.org/0000-0002-3399-6735

ORCID de J. L. Aparicio: https://orcid. org/0000-0002-8019-8103
ORCID de R. Beribé: https://orcid.org/0000-0001-6155-9332 ORCID de O. Patiño: https://orcid.org/0000-0002-7841-4081 


\section{BIBLIOGRAFÍA}

1. Tang X, Wang S, Zhan S, Niu J, Tao K, Zhang Y, et al. The prevalence of symptomatic knee osteoarthritis in China: results from the China Health and Retirement Longitudinal Study. Arthritis Rheumatol 2016;68(3):648-53. https://doi.org/10.1002/art.39465

2. Plotnikoff R, Karunamuni N, Lytvyak E, Penfold C, Schopflocher D, Imayama I, et al. Osteoarthritis prevalence and modifiable factors: a population study. BMC Public Health 2015;15:1195.

https://doi.org/10.1186/s12889-015-2529-0

3. Dillon CF, Rasch EK, Gu Q, Hirsch R. Prevalence of knee osteoarthritis in the United States: arthritis data from the Third National Health and Nutrition Examination Survey 1991-94. J Rheumatol 2006;33(11):2271-9. PMID: 17013996

4. Turkiewicz A, Gerhardsson de Verdier M, Engström G, Nilsson PM, Mellström C, Lohmander LS, et al. Prevalence of knee pain and knee OA in southern Sweden and the proportion that seeks medical care. Rheumatology (Oxford) 2015;54(5):827-35. https://doi.org/10.1093/rheumatology/keu409

5. Castell MV, van der Pas S, Otero A, Siviero P, Dennison E, Denkinger M, et al. Osteoarthritis and frailty in elderly individuals across six European countries: results from the European Project on OSteoArthritis (EPOSA). BMC Musculoskelet Disord 2015;16:359. https://doi.org/10.1186/s12891-015-0807-8

6. National Institutes of Health Consensus Program. NIH Consensus Development Conference on Total Knee Replacement - Final Statement. Disponible en:

https://consensus.nih.gov/2003/2003totalkneereplacement117html.htm

7. Lizaur A, Marco L, Cebrian R. Preoperative factors influencing the range of movement after total knee arthroplasty for severe osteoarthritis. J Bone Joint Surg Br 1997;79(4):626-9. https://doi.org/10.1302/0301-620x.79b4.7242

8. Farahini H, Moghtadaei M, Bagheri A, Akbarian E. Factors influencing range of motion after total knee arthroplasty. Iran Red Crescent Med J 2012;14(7):417-21. PMID: 22997557

9. Naili JE, Iversen MD, Esbjörnsson AC, Hedström M, Schwartz MH, Häger CK, et al. Deficits in functional performance and gait one year after total knee arthroplasty despite improved self-reported function. Knee Surg Sports Traumatol Arthrosc 2017;25(11):3378-86. https://doi.org/10.1007/s00167-016-4234-7

10. Mizner RL, Petterson SC, Clements KE, Zeni JA Jr, Irrgang JJ, Snyder-Mackler L. Measuring functional improvement after total knee arthroplasty requires both performance-based and patient-report assessments: a longitudinal analysis of outcomes. J Arthroplasty 2011;26(5):728-37. https://doi.org/10.1016/j.arth.2010.06.004

11. Bade MJ, Kohrt WM, Stevens-Lapsley JE. Outcomes before and after total knee arthroplasty compared to healthy adults. J Orthop Sports Phys Ther 2010;40(9):559-67. https://doi.org/10.2519/jospt.2010.3317

12. Ransen M, Nairn L, Bridgett L, Crosbie J, March L, Parker D, et al. Post-acute rehabilitation after total knee replacement: a multicenter randomized clinical trial comparing long-term outcomes. Arthritis Care Res 2017;69:192-200. https://doi.org/10.1002/acr.23117

13. Ishii Y, Noguchi H, Sato J, Sakurai T, Toyabe SI. Quadriceps strength impairment in the mid- to long-term followup period after total knee arthroplasty. Knee Surg Sports Traumatol Arthrosc 2017;25(11):3372-7. https://doi.org/10.1007/s00167-016-4333-5

14. Kievit AJ, van Geenen RC, Kuijer PP, Pahlplatz TM, Blankevoort L, Schafroth MU. Total knee arthroplasty and the unforeseen impact on return to work: a cross-sectional multicenter survey. J Arthroplasty 2014;29(6):1163-8. https://doi.org/10.1016/j.arth.2014.01.004

15. Postel JM, Thoumie P, Missaoui B, Biau D, Ribinik P, Revel M, Rannou F; French Physical Medicine and Rehabilitation Society. Continuous passive motion compared with intermittent mobilization after total knee arthroplasty. Elaboration of French clinical practice guidelines. Ann Readapt Med Phys 2007;50(4):244-57. https://doi.org/10.1016/j.annrmp.2007.03.001

16. O'Driscoll SW, Giori NJ. Continuous passive motion (CPM): theory and principles of clinical application. J Rehabil Res Dev 2000;37(2):179-88. PMID: 10850824

17. Harvey LA, Brosseau L, Herbert RD. Continuous passive motion following total knee arthroplasty in people with arthritis. Cochrane Database Syst Rev 2010;(2):CD004260. https://doi.org/10.1002/14651858.CD004260.pub3

18. Brosseau L, Milne S, Wells G, Tugwell P, Robinson V, Casimiro L, et al. Efficacy of continuous passive motion following total knee arthroplasty: a metaanalysis. J Rheumatol 2004;31(11):2251-64. PMID: 15517640 
19. Maniar RN, Baviskar JV, Singhi T, Rathi SS. To use or not to use continuous passive motion post-total knee arthroplasty presenting functional assessment results in early recovery. J Arthroplasty 2012;27(2):193-200.e1. https://doi.org/10.1016/j.arth.2011.04.009

20. Alkire MR, Swank ML. Use of inpatient continuous passive motion versus no CPM in computer-assisted total knee arthroplasty. Orthop Nurs 2010;29(1):36-40. https://doi.org/10.1097/NOR.0b013e3181c8ce23

21. Lenssen AF, Crijns YH, Waltjé EM, Roox GM, van Steyn MJ, Geesink RJ, et al. Effectiveness of prolonged use of continuous passive motion (CPM) as an adjunct to physiotherapy following total knee arthroplasty: design of a randomised controlled trial [ISRCTN85759656]. BMC Musculoskelet Disord 2006;7:15. https://doi.org/10.1186/1471-2474-7-15

22. Bruun-Olsen V, Heiberg KE, Mengshoel AM. Continuous passive motion as an adjunct to active exercises in early rehabilitation following total knee arthroplasty - a randomized controlled trial. Disabil Rehabil 2009;31(4):277-83. https://doi.org/10.1080/09638280801931204

23. Denis M, Moffet H, Caron F, Ouellet D, Paquet J, Nolet L. Effectiveness of continuous passive motion and conventional physical therapy after total knee arthroplasty: a randomized clinical trial. Phys Ther 2006;86(2):17485. PMID: 16445331

24. Joshi RN, White PB, Murray-Weir M, Alexiades MM, Sculco TP, Ranawat AS. Prospective randomized trial of the efficacy of continuous passive motion post total knee arthroplasty: experience of the Hospital for Special Surgery. J Arthroplasty 2015;30(12):2364-9. https://doi.org/10.1016/j.arth.2015.06.006

25. Chen LH, Chen CH, Lin SY, Chien SH, Su JY, Huang CY, et al. Aggressive continuous passive motion exercise does not improve knee range of motion after total knee arthroplasty. J Clin Nurs 2013;22(3-4):389-94. https://doi.org/10.1111/j.1365-2702.2012.04106.x

26. Boese CK, Weis M, Phillips T, Lawton-Peters S, Gallo T, Centeno L. The efficacy of continuous passive motion after total knee arthroplasty: a comparison of three protocols. J Arthroplasty 2014;29(6):1158-62. https://doi.org/10.1016/j.arth.2013.12.005

27. Worland RL, Arredondo J, Angles F, Lopez-Jimenez F, Jessup DE. Home continuous passive motion machine versus professional physical therapy following total knee replacement. J Arthroplasty 1998;13(7):784-7. https://doi.org/10.1016/s0883-5403(98)90031-6

28. Lotke PA, Faralli VJ, Orenstein EM, Ecker ML. Blood loss after total knee replacement. Effects of tourniquet release and continuous passive motion. J Bone Joint Surg Am 1991;73(7):1037-40. PMID: 1874765

29. Pope RO, Corcoran S, McCaul K, Howie DW. Continuous passive motion after primary total knee arthroplasty. Does it offer any benefits? J Bone Joint Surg Br 1997;79(6):914-7. https://doi.org/10.1302/0301-620x.79b6.7516

30. Pua YH1, Ong PH, Chong HC, Yeo W, Tan C, Lo NN. Knee extension range of motion and self-report physical function in total knee arthroplasty: mediating effects of knee extensor strength. BMC Musculoskelet Disord 2013;14:33. https://doi.org/10.1186/1471-2474-14-33

31. Holm B, Kristensen MT, Bencke J, Husted H, Kehlet H, Bandholm T. Loss of knee-extension strength is related to knee swelling after total knee arthroplasty. Arch Phys Med Rehabil 2010;91(11):1770-6. https://doi.org/10.1016/j.apmr.2010.07.229

32. Escobar A, Quintana JM, Bilbao A, Azkárate J, Güenaga JI. Validation of the Spanish version of the WOMAC questionnaire for patients with hip or knee osteoarthritis. Western Ontario and McMaster Universities Osteoarthritis Index. Clin Rheumatol 2002;21(6):466-71. https://doi.org/10.1007/s100670200117

33. Podsiadlo D, Richardson S. The timed "Up \& Go": a test of basic functional mobility for frail elderly persons. J Am Geriatr Soc 1991;39(2):142-8. https://doi.org/10.1111/j.1532-5415.1991.tb01616.x

34. Suri S, Gill SE, Massena de Camin S, Wilson D, McWilliams DF, Walsh DA. Neurovascular invasion at the osteochondral junction and in osteophytes in osteoarthritis. Ann Rheum Dis 2007;66(11):1423-8. https://doi.org/10.1136/ard.2006.063354

35. Goldring SR, Goldring MB. Changes in the osteochondral unit during osteoarthritis: structure, function and cartilage-bone crosstalk. Nat Rev Rheumatol 2016;12(11):632-44. https://doi.org/10.1038/nrrheum.2016.148

36. Stevens-Lapsley JE, Balter JE, Wolfe P, Eckhoff DG, Kohrt WM. Early neuromuscular electrical stimulation to improve quadriceps muscle strength after total knee arthroplasty: a randomized controlled trial. Phys Ther 2012;92(2):210-26. https://doi.org/10.2522/ptj.20110124 
37. Petterson SC, Mizner RL, Stevens JE, Raisis L, Bodenstab A, Newcomb W, et al. Improved function from progressive strengthening interventions after total knee arthroplasty: a randomized clinical trial with an imbedded prospective cohort. Arthritis Rheum 2009;61(2):174-83. https://doi.org/10.1002/art.24167

38. Bade MJ, Kittelson JM, Kohrt WM, Stevens-Lapsley JE. Predicting functional performance and range of motion outcomes after total knee arthroplasty. Am J Phys Med Rehabil 2014;93(7):579-85. https://doi.org/10.1097/PHM.0000000000000065

39. Palmieri-Smith RM, Villwock M, Downie B, Hecht G, Zernicke R. Pain and effusion and quadriceps activation and strength. J Athl Train 2013;48(2):186-91. https://doi.org/10.4085/1062-6050-48.2.10

40. Pietrosimone BG, Hertel J, Ingersoll CD, Hart JM, Saliba SA. Voluntary quadriceps activation deficits in patients with tibiofemoral osteoarthritis: a meta-analysis. PM R 2011;3(2):153-62; quiz 162. https://doi.org/10.1016/j.pmrj.2010.07.485

41. Rice DA, McNair PJ. Quadriceps arthrogenic muscle inhibition: neural mechanisms and treatment perspectives. Semin Arthritis Rheum 2010;40(3):250-66. https://doi.org/10.1016/j.semarthrit.2009.10.001

42. Herbold JA, Bonistall K, Blackburn M, Agolli J, Gaston S, Gross C, et al. Randomized controlled trial of the effectiveness of continuous passive motion after total knee replacement. Arch Phys Med Rehabil 2014;95(7):1240-5. https://doi.org/10.1016/j.apmr.2014.03.012 